\title{
Engineering of daidzein 3'-hydroxylase P450 enzyme into catalytically self-sufficient cytochrome P450
}

\author{
Kwon-Young Choi ${ }^{1}$, EunOk Jung ${ }^{1}$, Da-Hye Jung ${ }^{1}$, Byeo-Ri An ${ }^{1}$, Bishnu Prasad Pandey ${ }^{1}$, Hyungdon Yun²,
} Changmin Sung ${ }^{3}$, Hyung-Yeon Park ${ }^{4^{*}}$ and Byung-Gee Kim ${ }^{1^{*}}$

\begin{abstract}
A cytochrome P450 (CYP) enzyme, 3'-daidzein hydroxylase, CYP105D7 (3'-DH), responsible for daidzein hydroxylation at the 3'-position, was recently reported. CYP105D7 (3'-DH) is a class I type of CYP that requires electrons provided through electron transfer proteins such as ferredoxin and ferredoxin reductase. Presently, we constructed an artificial CYP in order to develop a reaction host for the production of a hydroxylated product. Fusion-mediated construction with the reductase domain from self-sufficient CYP102D1 was done to increase electron transfer efficiency and coupling with the oxidative process. An artificial self-sufficient daidzein hydroxylase (3'-ASDH) displayed distinct spectral properties of both flavoprotein and CYP. The fusion enzyme catalyzed hydroxylation of daidzein more efficiently, with a $k_{\text {cat }} / K_{m}$ value of $16.8 \mu \mathrm{M}^{-1} \mathrm{~min}^{-1}$, which was about 24-fold higher than that of the 3'-DH-camA/B reconstituted enzyme. Finally, a recombinant Streptomyces avermitilis host for the expression of 3'-ASDH and production of the hydroxylated product was developed. The conversion that was attained (34.6\%) was 5.2-fold higher than that of the wild-type.
\end{abstract}

Keywords: Cytochrome P450 monooxygenases, Daidzein 3'-hydroxylase, Self-sufficient P450, CYP102D1

\section{Introduction}

Cytochrome P450 monooxygenases (CYPs) are a superfamily of heme-thiolate containing enzymes that catalyze a variety of chemical reactions in regio/stereo-selective manners [1]. Various types of reactions catalyzed by CYP have been identified; generally, these enzymes act as monooxygenases that catalyze the introduction of an oxygen molecule into the substrate whereby one atom of molecular oxygen is incorporated into the product [2]. Oxidation is one of the most valuable reactions in modern industrial processes involving drug development and bio-fuel generation, and is the most common P450catalyzed reaction for both endogenous and exogenous compounds $[3,4]$.

\footnotetext{
*Correspondence: hypark@snu.ac.kr; byungkim@snu.ac.kr

${ }^{1}$ School of Chemical and Biological Engineering, Institute of Bioengineering, Seoul National University, Seoul, South Korea

${ }^{4}$ Korea Bio-Hub Center, Bio-MAX Institute, Seoul National University, Seoul, South Korea

Full list of author information is available at the end of the article
}

Fundamentally, the CYP-catalyzed oxidation reaction requires the sequential input of two electrons and two protons $\left([\mathrm{P} 450-\mathrm{RH}]+2 \mathrm{e}^{-}+2 \mathrm{H}^{+}+\mathrm{O}_{2} \rightarrow \mathrm{P} 450+\mathrm{ROH}+\right.$ $\mathrm{H}_{2} \mathrm{O}$ ) in the presence of oxygen to activate a R-H bond, followed by the release of the hydroxylated product, R$\mathrm{OH}$, and water [5]. CYPs require a tight interaction with auxiliary proteins, electron transferring proteins from a cofactor. The combination and orientation of redox proteins classifies CYPs into several types [6-8]. Among them, especially, the class III cytochrome P450 system consists of a self-sufficient CYP, in which the heme domain is fused with a P450 reductase domain and encoded in a single polypeptide $[9,10]$. Several self-sufficient P450s belonging to the CYP102A family, as well as CYP102D1, have been identified from the genome sequences of various Bacillus species such as CYP102A2 and CYP102A3 from Bacillus subtilis, CYP102A5 from B. cereus and CYP102A7 from B. licheniformis, and recently, CYP102D1 was identified from Streptomyces avermitilis [11-15]. Especially, the fusion arrangement as self-sufficient CYPs enhances catalytic efficiency, in

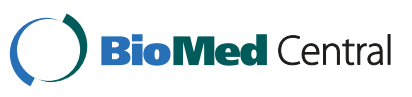


terms of the kinetic parameters $k_{\text {cat }}$ and $\mathrm{K}_{\mathrm{m}}$ [16]. For example, CYP102A1 BM3 catalyses fatty acid hydroxylation at rates of up to approximately $17,000 \mathrm{~min}^{-1}$, which is at least two orders of magnitude faster than observed for other class I type fatty acid hydroxylases [6,17]. The knowledge that self-sufficient P450s exhibit the highest turnover frequency due to the higher possibility of contact between the heme and reductase domains, and the induction of intra-molecular electron transfer, have prompted several attempts to make an artificial selfsufficient system by fusing redox proteins. In one study, the N-terminally modified human P450s CYP2C9, CYP2C19 and CYP3A4 fused to the soluble NADPHdependent oxidoreductase domain of CYP102A1 were constructed to exploit the advantages of the fused nature of the bacterial CYP102A1 system [18]. Also, P450cam from Pseudomonas putida (CYP101A) and P450bzo from an environmental metagenome library (CYP203A) were fused with the reductase domain of self-sufficient P450RhF from Rhodococcus sp. NCIMB 9784 to increase catalytic efficiency [19].

Recently our group reported two enzymes, CYP105D7 (3'-DH), which is especially responsible for daidzein hydroxylation at the 3'-position of the daidzein B-ring with very high regio-selectivity, and CYP102D1, a unique self-sufficient P450 from S. avermitilis $[15,20]$. The 3'-DH encoded by sav7469 from $S$. avermitilis catalyzes the hydroxylation of daidzein to produce 3,4,7trihydroxyisoflavone (3'-ODI) and is a class I CYP, which are necessary electron transfer proteins. CYP102D1 is also a promising model for the construction of an artificial self-sufficient CYP with 3'-DH. Here, we demonstrate the fusion-mediated arrangement of 3'-DH that was achieved by engineering a class I type of CYP into an artificial self-sufficient CYP to increase enzyme catalytic activity and the bio-conversion of daidzein (Figure 1).

Furthermore, P450 systems are especially welldeveloped in Streptomyces strains producing various kinds of secondary metabolites, such as antibiotics that are in most cases regulated by $\mathrm{P} 450$ systems at the last step. Many of these systems bestow potent biological activities [21]. Especially, S. avermitilis produces 33 P450 enzymes that are valuable as extra- or intra-cellular enzymes capable of catalyzing targeting molecules, as well as more than half of the known biologically active microbial products, including many commercially important antibiotics, immunosuppressive compounds, animal health products and agrochemicals [22]. This vast reservoir of diverse products has made $S$. avermitilis one of the most important industrial microbial genera. A battery of tool for the genetic manipulation of the organism is available [23]. Presently, with the goal of constructing a versatile model host for the biotransformation of daidzein by the heterologous expression of CYP enzymes, the 3'-ASDH gene was amplified with and without 3'-DH gene. The recombinant $S$. avermitilis strains proved to be a suitable natural host for the expression of this artificial fusion enzyme.

\section{Material and methods Chemicals}

Daidzein (7,4'-dihydroxyisoflavone) and 3,4,7-trihydroxyisoflavone (3'-ODI) used in this study were donated by the Skin Research Institute, Amorepacific R\&D Center, South Korea. N,O-bis(trimethylsily)trifluoroacetamide used for derivatization before gas chromatography/mass spectrometry (GC/MS) analyses was obtained from Fluka (Buchs, Switzerland). All other chemicals were of the highest grade available.

\section{Bacterial strain and culture condition}

S. avermitilis MA4680 was obtained from the Korea Collection for Type Cultures (KCTC; Daejeon, South Korea). The strain was cultivated on R2YE medium containing $10.3 \%$ sucrose, $1 \%$ glucose, $1 \% \mathrm{MgCl}_{2} \quad 6 \mathrm{H}_{2} \mathrm{O}$, $0.025 \% \mathrm{~K}_{2} \mathrm{SO}_{4}, 0.5 \%$ yeast extract, $0.01 \%$ casamino acid, $0.57 \%$ TES, $\quad 0.005 \% \mathrm{~K}_{2} \mathrm{HPO}_{4}, \quad 0.03 \% \quad \mathrm{CaCl}_{2} .2 \mathrm{H}_{2} \mathrm{O}$, $0.003 \% \mathrm{~L}$-proline, $2 \mathrm{ml}$ of trace element solution and $5 \mathrm{~mL}$ of $1 \mathrm{~N} \mathrm{NaOH}$. Seed culture of $S$. avermitilis MA4680 was generated in test tubes containing $5 \mathrm{~mL}$ R2YE medium by shaking at $220 \mathrm{rpm}$ for 3 days at $28^{\circ} \mathrm{C}$. Serial subculture was performed in $50 \mathrm{~mL}$ of the aforementioned medium containing $50 \mu \mathrm{g}$ of thiostrepton for more than 3 days at $28^{\circ} \mathrm{C}$.
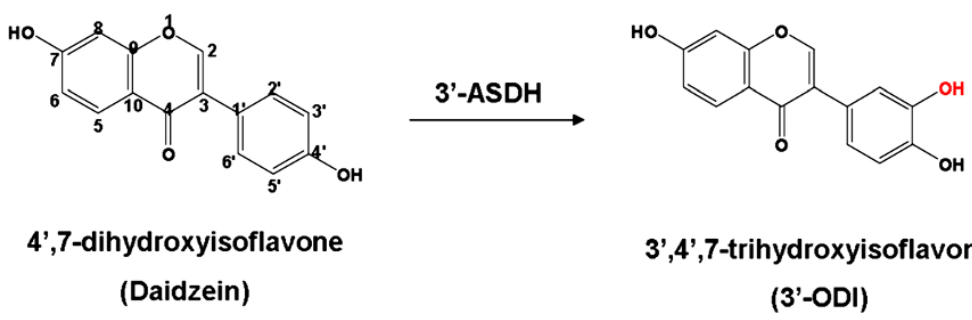

3',4',7-trihydroxyisoflavone (3'-ODI)

Figure 1 Chemical structure and oxidative metabolite of daidzein by 3'-ASDH. This artificial fusion enzyme catalyzes the hydroxylation at the 3'-position of daidzein B-ring with very high regio-selectivity. 


\section{Gene manipulation}

Chromosomal DNA from S. avermitilis MA4680 was prepared using G-spin ${ }^{\mathrm{TM}}$ for Bacteria Genomic DNA Extraction kit (iNtRON, Seungnam, South Korea). Plasmids from Escherichia coli strains were prepared using the GeneAll DNA Purification System (Geneall Biotechnology, Seoul, South Korea). All polymerase chain reaction (PCR) amplifications by were performed using Pfu polymerase with GC II buffer (Takara Bio, Shiga, Japan). Primers used in this study were commercially synthesized by Cosmo Bioscience (Cosmo, Seoul, South Korea). DNA of CYP105D7 and CYP102D1 with codon optimized to suit the codon preference bias of $E$. coli were fully synthesized by Bioneer (Daejeon, South Korea).

To construct the plasmid for the $S$. avermitilis system, Streptomyces expression vector pIBR25 was used. Three sets of over-expressing plasmids (pIBR25::3'-DH, pIBR25::3'-ASDH and pIBR $\Delta 3$ '-DH::3'-ASDH) and one disruption plasmid (pIBR $\Delta 3^{\prime}-\mathrm{DH}$ ) were constructed. For each set of primer designs an extra 50 nucleotides harboring the ribosomal binding site region was obtained from the upstream region of the gene. The 3'-DH gene was disrupted in $S$. avermitilis through homologous recombination. To inactivate 3'-DH, the $2.5 \mathrm{~kb}$ upstream and $2.5 \mathrm{~kb}$ downstream fragments were amplified together with the apramycin resistance gene in the pUC19 vector. Plasmids were digested with restriction enzymes and ligated into the Supercos1 vector. Transformants carrying the single and double crossovers were distinguished by replica plaiting [24]. Finally, four recombinant strains were prepared for the evaluation of daidzein biotransformation by 3'-ASDH.

\section{Artificial self-sufficient daidzein hydroxylase construction by fusion arrangement and construction of recombinant S. Avermitilis expressing 3'-ASDH}

The reductase domain of CYP102D1 including the liker domain (VRARQEHERTVFGAADLQTD) was PCRamplified from a synthetic CYP102D1 gene using a primer set. The PCR reaction conditions consisted of 30 cycles of denaturation for $1 \mathrm{~min}$ at $94{ }^{\circ} \mathrm{C}$, annealing for $1 \mathrm{~min}$ at $68{ }^{\circ} \mathrm{C}$ and extension for $1.5 \mathrm{~min}$ at $72{ }^{\circ} \mathrm{C}$. The PCR product was cloned into the pET24ma(+) expression vector (Pasteur Institute, Paris, France) using the restriction enzymes SacI and HindIII. The heme domain (from Met1 to Thr402) of CYP105D7 which was chemically synthesized after codon optimization complying with the codon preferences of $E$. coli were digested with EcoRI and SacI restriction enzymes (Bioneer, Seoul, Korea). Correctly assembled DNA was cloned into the pET-24ma(+) harboring reductase domain (from Asp478 to Ala1073) of CYP102D1.

To transform the protoplasts $S$. avermitilis, a previously detailed polyethylene glycol (PEG)-associated transformation method was used [25]. The plasmid $(1 \mu \mathrm{g} / \mu \mathrm{L}$ in $\mathrm{TE})$ was added to $0.1 \mathrm{~mL}$ of a protoplast suspension containing approximately $10^{2}$ protoplasts generated by a 15 -min lysozyme treatment. Five hundred microliters of the protoplast buffer containing 30\% (w/v) PEG1000 (Sigma-Aldrich, St. Louis, MO) was added to the mixture, which was carefully pipetted up-and-down. This protoplast suspension was centrifuged after addition of $300 \mu \mathrm{L}$ of the protoplast buffer. The suspension was plated into a regeneration medium by gentle shaking. After 16-18 h incubation, the plate was overlaid with $10 \mu \mathrm{g} / \mathrm{mL}$ of thiostrepton.

\section{Expression and purification of 3 '-ASDH and its ultraviolet/ visible spectral features}

E. coli BL21(DE3) cells containing 3'-ASDH was cultured in Luria-Bertani (LB) medium containing $50 \mu \mathrm{g} /$ $\mathrm{mL}$ of kanamycin at $37{ }^{\circ} \mathrm{C}$ until the cell concentration reached an optical density at $600 \mathrm{~nm}\left(\mathrm{OD}_{600}\right)$ of 0.6 , isopropyl-thio- $\beta$-D-galactopyranoside (IPTG) and $\delta$ aminolevlunic acid as heme precursor were added to a final concentration of $0.25 \mathrm{mM}$, followed by growing for overnight at $30{ }^{\circ} \mathrm{C}$. Bacteria were harvested by centrifugation and washed twice with phosphate buffered saline (PBS), resuspended in $5 \mathrm{~mL}$ of a sonication buffer $(\mathrm{pH}$ 7.0) and disrupted by sonication. Disrupted soluble fractions were collected by centrifugation and purified using a Ni-NTA his-tag purification kit (QIAGEN Korea Ltd, Seoul, South Korea). Purification was carried out utilizing the Ni-NTA resin. The Ni-NTA bound enzymes were washed twice with $50 \mathrm{mM}$ potassium phosphate buffer ( $\mathrm{pH} 7.0$ ) containing $500 \mathrm{mM} \mathrm{NaCl}$ and $50 \mathrm{mM}$ imidazole, and enzymes were eluted with the same buffer containing $200 \mathrm{mM}$ imidazole. Finally, imidazole and sodium chloride were removed by dialysis against $50 \mathrm{mM}$ potassium phosphate buffer ( $\mathrm{pH}$ 7.0). Purified proteins were subjected to $10 \%$ sodium dodecyl sulfatepolyacrylamide gel electrophoresis (SDS-PAGE) and spectrophotometric analysis to measure carbon monoxide (CO)-binding activity [26]. Absorption spectra of CO-bound recombinant CYP proteins after sodium dithionite reduction were measured by UV/vis spectrometry (Thermo Lab Systems, Beverly, MA, USA) by scanning wavelength from 400-500 nm [26]. The protein concentration was estimated using reduced $\mathrm{CO}$ versus reduced difference spectra. The 3'-ASDH protein content was determined using an extinction coefficient of $91.9 \mathrm{mM}^{-1} \mathrm{~cm}^{-1}$ at $450 \mathrm{~nm}$ [26].

\section{Determination of kinetic values of $3^{\prime}$-ASDH}

NADPH oxidation was measured by the absorption decrease at $340 \mathrm{~nm}$ with UV/vis spectrometry (Thermo Lab Systems, Beverly, MA, USA). A solution containing $160 \mu \mathrm{L}$ of potassium phosphate buffer ( $\mathrm{pH} 7.5), 10 \mu \mathrm{L}$ of 
each substrate concentration solution in dimethyl sulfoxide (DMSO) and $20 \mu \mathrm{L}$ of enzyme solution $(10 \mu \mathrm{mol} /$ $\mathrm{mL}$ ) was incubated for $5 \mathrm{~min}$ in a 96-well microplate. The reaction was started by adding $10 \mu \mathrm{L}$ of a $10 \mathrm{mM}$ $\mathrm{NADPH}$ solution. For determination of kinetic parameters, a substrate concentration range of $2.5-50 \mu \mathrm{M}$ of daidzein was used. All data were fitted to the MichaelisMenten equation by linear regression. As a reference sample, NADPH consumption rates were measured in the absence of substrate.

\section{Substrate binding spectra of each CYP ( $\mathrm{K}_{\mathrm{d}}$ value)}

Spectroscopic substrate binding spectra were obtained as described previously [17]. A solution of substrate was added to the enzyme solution to produce a final substrate concentration of $2.5-100 \mu \mathrm{M}$. For the determination of $K_{d}$ values, the reciprocal values of the absorption difference between $394 \mathrm{~nm}$ and $418 \mathrm{~nm}$ were plotted against the reciprocal values of the substrate concentration. For calculation of the $K_{d}$ value of the enzyme-substrate, the experimental data were fitted to a hyperbola curve $\left(y=a x(b+x)^{-1}\right)$ by a nonlinear regression procedure based on the Durbin-Watson algorithm in SigmaPlot software (Systat, San Diego, CA).

\section{Resting cell assay of E. Coli BL21(DE3) expressing 3'-ASDH in the bioconversion of daidzein}

Cells were harvested and washed twice with $50 \mathrm{~mL}$ of PBS buffer. And the cells were resuspended in potassium phosphate buffer (100 mM, pH 7.0) containing $1 \%$ of glucose. The initial substrate stock solution was prepared by dissolving daidzein $(24.5 \mathrm{mg})$ into the DMSO and diluted to reaction concentration $(100 \mu \mathrm{M})$ using DMSO: $\mathrm{MeOH}$ (50:50) solvent. Whole cell reactions were initiated by the addition of the substrate at $37{ }^{\circ} \mathrm{C}$ in a shaker $(200 \mathrm{rpm})$ for $24 \mathrm{~h}$. The reaction was quenched by the addition of same volume of ethyl acetate to the reaction, followed by vigorous vortexing. The mixtures were centrifuged at 13,000 rpm for $10 \mathrm{~min}$, after which the upper layer containing any remaining substrate and products (oraganic layer) was extracted, followed by evaporation of the supernatant using a vacuum (BioTron, Bucheon, South Korea). The sample was subsequently used for GC/MS structural and quantitative analyses.

\section{Resting cell assay of recombinant S. Avermitilis in the bioconversion of daidzein}

Each recombinant $S$. avermitilis cells harvested from $50 \mathrm{~mL}$ of culture broth were washed twice with potassium phosphate buffer $(50 \mathrm{mM}, \mathrm{pH} 7.2)$. After centrifugation (13,000 rpm, $10 \mathrm{~min}$ ), $5 \mathrm{~g}$ of cells (wet wt.) were added to $50 \mathrm{~mL}$ of potassium phosphate buffer (100 mM, pH 7.2) with $0.1 \mathrm{mM}$ of final substrate concentration in DMSO:MeOH (50:50). The mixture containing $50 \mathrm{~mL}$ in $250 \mathrm{~mL}$ flask was incubated in a shaker at $200 \mathrm{rpm}$ for $72 \mathrm{~h}$ at $28{ }^{\circ} \mathrm{C}$ and the reaction mixture was extracted with ethylacetate (JUNSEI, Japan). The extracted sample was evaporated in a centrifugal vacuum concentrator (BioTron, South Korea). And the solvents in DMSO:MeOH (50:50) was added in the reaction with the final volumetric ratio from $0 \%$ to $40 \%$ in order to examine the biotransformation conversion depending on organic solvent concentrations.

\section{GC/MS analysis of products}

For structural analysis of oxidative metabolites of daidzein, samples extracted with ethylacetate were evaporated to dryness and dissolved in $100 \mu \mathrm{L}$ methanol. The same volume of $0.5 \mathrm{M} \mathrm{NH}_{4} \mathrm{CO}_{3}$ and $2 \mathrm{M} \mathrm{KCl}$ was added to each sample, and the mixture was centrifuged at room temperature, after which the supernatant was transferred to a new vial for derivatization. To protect hydroxyl groups, reaction products were derivatized using N,O-bis(trimethylsilyl)trifluoroacetamide (BSTFA) by heating at $60{ }^{\circ} \mathrm{C}$ for $60 \mathrm{~min}$. GC/MS was performed using a Finnigan MAT system (Gas chromatograph model GCQ, HP 19091j-433; Thermo Labsystems, USA) connected to an ion trap mass detector. The BSTFA derivatives were separated through a non-polar capillary column $(5 \%$ phenyl methyl siloxane capillary $30 \mathrm{~m} \times 250 \mathrm{~nm}$ i.d., $0.24 \mu \mathrm{m}$ film thickness, HP-5) containing a linear temperature gradient (for fatty acids: at $100{ }^{\circ} \mathrm{C} 0.5 \mathrm{~min}, 5{ }^{\circ} \mathrm{C} / \mathrm{min}$ to $200{ }^{\circ} \mathrm{C}$, hold for $5 \mathrm{~min}$, $10{ }^{\circ} \mathrm{C} / \mathrm{min}$ to $250^{\circ} \mathrm{C}$, hold for $3 \mathrm{~min}$; for cyclic compounds: at $60{ }^{\circ} \mathrm{C} 1 \mathrm{~min}, 30{ }^{\circ} \mathrm{C} / \mathrm{min}$ to $250{ }^{\circ} \mathrm{C}$, hold for $10 \mathrm{~min}$, $1{ }^{\circ} \mathrm{C} / \mathrm{min}$ to $275^{\circ} \mathrm{C}$, hold for $3 \mathrm{~min}$ ). The injector port temperature was $250{ }^{\circ} \mathrm{C}$. Mass spectra were obtained by electron impact ionization at $70 \mathrm{eV}$, and scan spectra were obtained within the range of $100-600 \mathrm{~m} / \mathrm{z}$. Selected ion mode (SIM) was used for the detection and fragmentation analysis of major products.

\section{Homology modeling of $3^{\prime}$-ASDH}

Homology modeling of 3'-ASDH was performed by combinations of heme domain of CYP105D7 and reductase domain of CYP102D1. The homology model of CYP105D7 was constructed using 3ABB [27], a crystal structure of CYP105D6 involved in filipin hydroxylation, and CYP102D1 was constructed using 3hf2a, a crystal structure of CYP102A1 variant from the PDB database as a template. The predicted $3 \mathrm{D}$ structure was constructed based on a comparative homology modeling method using the Genefold and Composer programs from the SYBYL software package (Tripos Associates, St. Louis, MO). The initial state model was energyminimized by the conjugate gradient method at an energy gradient norm of $0.01 \mathrm{kcal} / \mathrm{mol}$. 


\section{Results}

Fusion-mediated self-sufficient construction of CYP105D7 with the reductase domain of CYP102D1

A recent study described the functional expression of a self-sufficient P450, CYP102D1, from S. avermitilis in E. coli [15]. Since the original gene coding CYP102D1 could not be successfully expressed in E. coli, the gene was chemically synthesized following codon optimization complying with the codon preferences of $E$. coli. The reductase domain consisting of the di-flavin binding domain (FAD/FMN) has linker sequences from Val458 and the coding synthetic DNA was first cloned into the expression vector pET24m(a). Target DNAs encoding daidzein hydroxylases were amplified with each specific primer and cloned into the pET24m(a)-CYP102D1 reductase (Figure 2). Especially, the sav7469 gene coding 3'-DH was not solely expressed in E. coli well; the codon optimized sav7469 gene missing stop codon based on $E$. coli codon bias was also used in the artificial selfsufficient P450 construction. Finally, the 3'-ASDH fusion protein was prepared by two synthetic DNA gene manipulations (Figure 2).

\section{Expression of artificial fusion proteins and their spectroscopic characterizations}

Figure 3A summarizes the expression of the constructed fusion protein. The protein was abundantly expressed as a soluble fraction yielding $19.2 \mathrm{mg} / \mathrm{L}$ of 3'-ASDH, and His-tag purified protein was prepared for in vitro

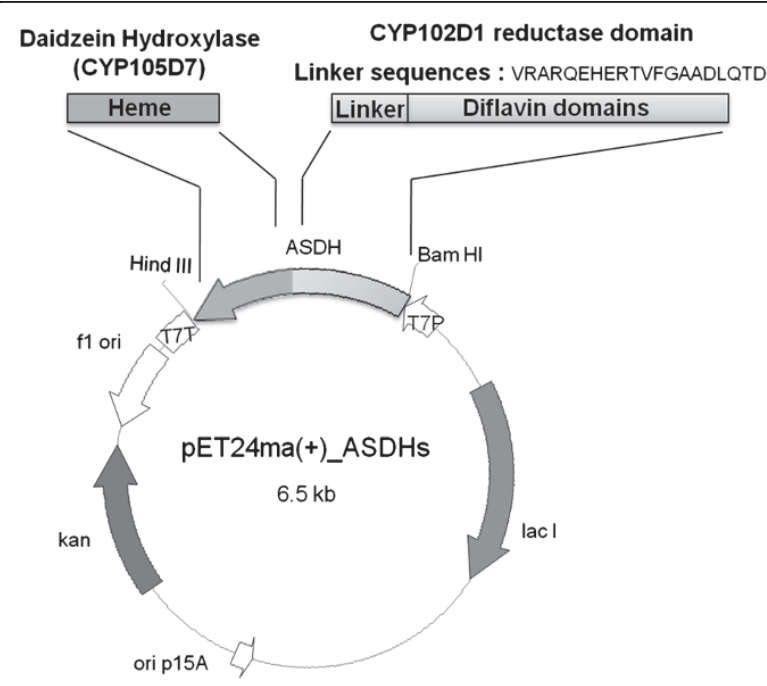

Figure 2 Design of artificial self-sufficient daidzein hydroxylases by heme domain swapping with self-sufficient CYP102D1 from Streptomyces avermitilis. The reductase domain consisting of di-flavin binding domain (FAD/FMN) has linker sequences from Val458 and the coding synthetic DNA was first cloned into the expression vector pET24m(a). And the target DNAs coding daidzein hydroxylases were amplified with each specific primer and coned into the pET24m(a)-CYP102D1 reductase. reaction as a reconstituted system. The purified fusion protein displayed a single protein band corresponding to a theoretical molecular weight of about $110 \mathrm{kDa}$, which was deduced by taking the reductase domain of CYP102D1 as $65 \mathrm{kDa}$, and finally concentrated $(\times 1500)$ to yield approximately $3.0 \mathrm{mg} / \mathrm{mL}(1.0 \mu \mathrm{M})$ (Figure $3 \mathrm{~A}$ ).

To examine the functional folding of the purified protein in the ferric state (reduced form), UV/vis spectroscopy was utilized. In general, a cysteine-thiolate ligand coordinated to the heme iron atom of CYP regulates any spectroscopic and catalytic properties. Figure 3B shows the absorption spectra of the fusion protein. Absorption spectroscopy of the purified artificial self-sufficient protein in its oxidized form (dashed line) and reduced form (dash-dotted line) revealed general features of bacterial CYPs. The oxidized form of spectra shows typical spectra of self-sufficient CYP enzyme with Soret peak at $419 \mathrm{~nm}$, an $\alpha$-band at $567 \mathrm{~nm}$, and and unresolved $\beta$ band due to the overlap with a broad shoulder in the 440-490 nm regison. This shoulder was interpreted as the flavin prosthetic groups. The 440-490 nm shoulder disappears upon addition of sodium dithionite owing to reduction of the flavins [28]. In addition, the absorption spectra of the reduced-CO oxidized forms are indicated in the inset. The CO-difference spectra resulting from the insertion of $\mathrm{CO}$ gas into a reduced state by sodium dithionite revealed that the heme Soret absorption maximum was at $450 \mathrm{~nm}$ [19]. As the reductase domain of CYP102D1 uses NADPH as an electron donating source, the constructed fusion enzyme used NADPH preferably (approximately $500 \mu \mathrm{mol}$ cyt $c / \mathrm{min} / \mu \mathrm{mol}$ CYP).

\section{Daidzein hydroxylation reaction catalyzed by 3 '-ASDH fusion enzyme}

The activity of 3'-ASDH fusion enzyme was measured using daidzein as a substrate in order to validate the efficiencies of fusion orientations with the natural threecomponent system. Previously, the CamA/B redox proteins from Pseudomonas putida were proven to be alternative electron transferring proteins [20]. Thus, the reconstituted 3'-DH and CamA/B enzyme activity was compared to that of 3'-ASDH. GC-MS assay of daidzein hydroxylation activity measured the hydroxylated products. The kinetic parameters of the 3'-ASDH enzyme were incredibly dominant over those of the reconstituted 3'-DH-camA/B system (Table 1), indicating that the fusion arrangement in a single polypeptide was absolutely efficient for inter-molecular electron transfer, rather than intra-molecular electron transfers, and was sufficient for CYP activity. As mentioned before, an excellent benefit of the fusion arrangement is that the covalent linkage presumably stabilizes the interaction between $\mathrm{P} 450$ and redox protein, enhancing electron transfer efficiency. As such, one would expect this to improve the catalytic 

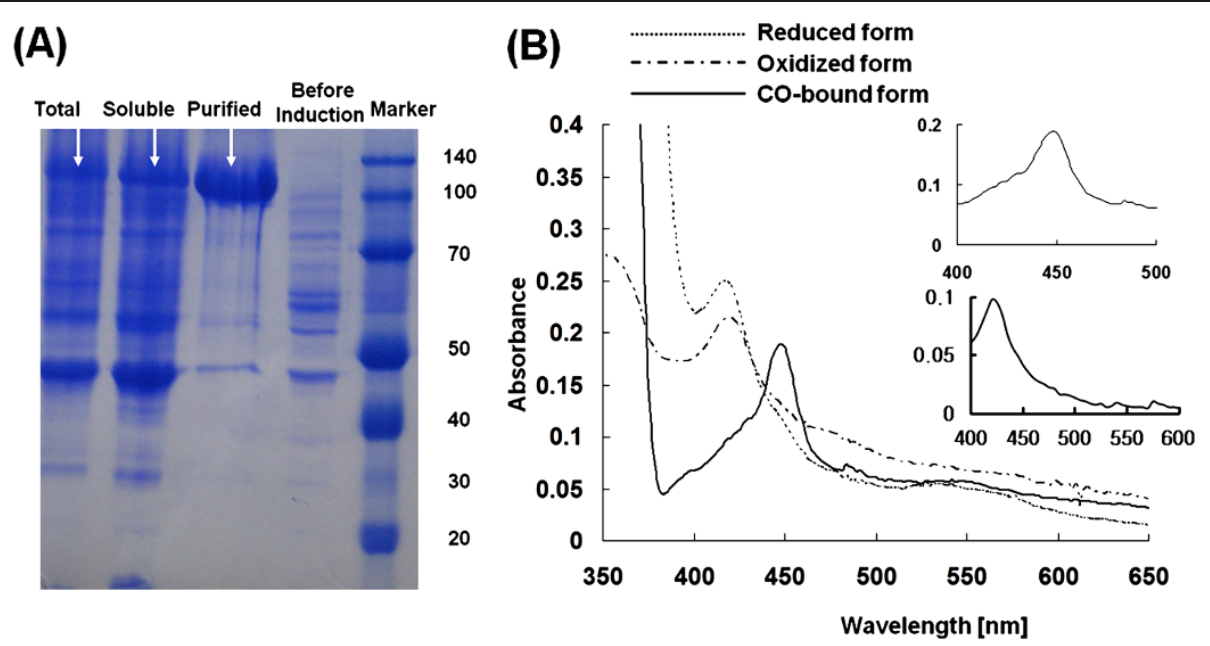

Figure 3 Expression and UV-visible absorption spectra of 3'-ASDH. (A) SDS/PAGE analysis of total cell protein content as well as purified protein containing the His-6 tag; the lanes, from the left, are total cell protein content after induction, total soluble protein content, purified protein content and total cell protein content before IPTG and protein marker, respectively. (B) UV-visible absorbance spectra of purified 3'-ASDH fusion protein revealed spectral features characteristic of CYPs; Dashed line: oxidized form, Dotted line: Reduced form with dithionite. Solid line: CO-difference spectra; Inserted figure: CO-difference spectra of 3'-ASDH.

activity in terms of $k_{\text {cat }}$, whereas the substrate specificity would not be changed significantly. The experimental results supported these expectations; the $K_{m}$ values of the reconstituted 3'-DH and 3'-ASDH proteins were quite similar in the range of approximately $20 \mu \mathrm{M}$, whereas the catalytic activity $\left(k_{c a t}\right)$ of the fused enzymes was significantly different from that of 3'-DH alone. The $k_{\text {cat }}$ values of 3'-ASDHs were approximately $342 \mathrm{nmol} /$ min- ${ }^{1} / \mathrm{nmol}$ of CYP, which is about 24 -fold higher than that of the reconstituted 3'-DH-camA/B system. These results indicate that the binding affinity $\left(K_{d}=15.3 \mu \mathrm{M}\right)$ between the heme domain of the $\mathrm{DH}$ enzyme and the substrate daidzein was not affected by fusion arrangement, whereas artificial fusion arrangements allowed effective intra-molecular electron transfer from NADPH to the heme domain through reductase, confirming our expectations. Effective electron transfer between CYP and reductase existing in different molecules of a fusion monooxygenase can occur if the two molecules are

Table 1 Comparison of kinetic parameters of P450s with natural redox partners and P450s fused to aself-sufficient mimic system

\begin{tabular}{lll}
\hline Kinetic parameters & $\begin{array}{l}\text { 3'-DH- }^{\prime} \text { CamA/B } \\
K_{m}[\mu M]\end{array}$ & 3'-ASDH \\
$k_{\text {cat }}$ & $21.8 \pm 3.6$ & $20.5 \pm 5.1$ \\
{$\left[\mathrm{~min}^{-1}\right]$} & $15.0 \pm 2.1$ & $342.3 \pm 53.6$ \\
$k_{c a t} / K_{m}$ & $0.7 \pm 0.4$ & $16.8 \pm 4.6$ \\
{$\left[\mu M^{-1} \mathrm{~min}^{-1}\right]$} & & \\
Coupling efficiency (\%) & $<55$ & $<72$ \\
\hline
\end{tabular}

dimeric partners, but this type of electron transfer cannot be discriminated from intramolecular transfer if the dissociation constant of the dimer is very small [15]. Such intramolecular electron transfer has been reported for naturally-occurring bacterial fusion CYP enzymes such as CYP102A family members catalyzing fatty acid hydroxylation.

Quantitative and structural analysis of reaction products using high-performance liquid chromatography (HPLC) and GC/MS

The resting cell assay using 3'-ASDH expressing E. coli BL21(DE3) revealed unique reaction products. The identification of oxidative metabolite was performed with GC/MS ${ }^{\mathrm{n}}$ (Figure 4A). Mass analysis of daidzein revealed a molecular ion mass of $398 \mathrm{~m} / \mathrm{z}$, and each monohydroxylated product was observed at $486 \mathrm{~m} / \mathrm{z}$, resulting in a $88 \mathrm{~m} / \mathrm{z}$ increase due to incorporation of molecular oxygen $(+16 \mathrm{~m} / \mathrm{z})$ after BSTFA derivatization $(+72 \mathrm{~m} / \mathrm{z})$ (Figure 4B). These values agree with the mass analysis patterns of the corresponding reference chemicals. The hydroxylated products were identified as 3'-ODI, which was mono-hydroxylated form of daidzein at the ortho position of 4'-hydroxyl group at the B-ring. Mass spectral analysis revealed that each had a molecular ion mass of $486 \mathrm{~m} / \mathrm{z}$ after BSTFA derivatization, which was exactly the same as those of the reference compounds. This result demonstrates that our previous understanding of daidzein hydroxylase reserves regio-selectivity after fusion arrangement with reductase domain regardless of catalytic activity for aromatic compounds such as ortho-hydroxylated daidzein. 


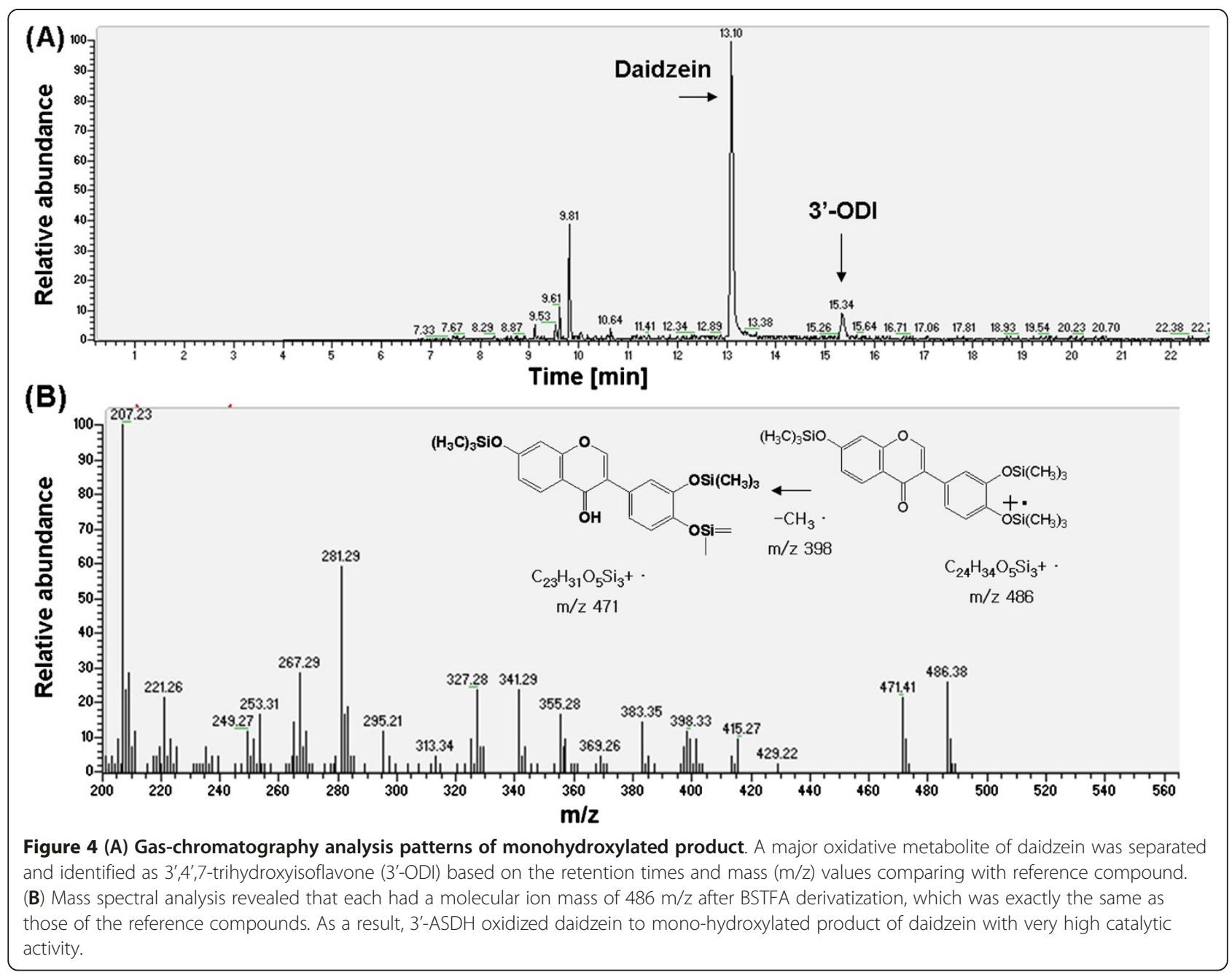

\section{Development of recombinant $S$.Avermitilis host system for heterologous expression of 3'-ASDH}

The resting cell assay in E. coli revealed that 3'-ASDH expressed in $E$. coli did not produce the expected conversion (approximately 5\%) when $100 \mu \mathrm{M}$ of daidzein was used as an initial substrate concentration, although purified 3'-ASDH enzyme showed very high catalytic activity.

Previously we reported that $S$. avermitilis strain is highly active and regiospecific to daidzein and genistein, producing monohydroxylated products $[20,29,30]$. The sav7469 gene encoding 3'-DH, among the 33 CYPs of S. avermitilis, dominantly catalyzed the regio-specific hydroxylation of daidzein to produce 3'-ODI. These results suggested that $S$. avermitilis is a very good reaction host for daidzein biotransformation by virtue of having the genomic encoding capacity for 3'-DH and its natural redox proteins. Thus, we presently sought the heterogeneous expression of 3'-ASDH in S. avermitilis and biotransformation of daidzein using recombinant strains.
Four recombinant $S$. avermitilis strains were constructed to compare the productivity of 3'-ODI, S. avermitilis $\Delta 3^{\prime}-\mathrm{DH}, \quad S$. avermitilis $\Delta 3^{\prime}-\mathrm{DH}:: 3^{\prime}-\mathrm{ASDH}, \quad S$. avermitilis::3'-DH and S. avermitilis:: 3'-ASDH (Table 2). As the $S$. avermitilis wild-type genome encodes 3'-DH (sav7469), the wild-type alone could convert daidzein into 3'-ODI at a rate of $6.7 \%$. After deletion of the sav7469 gene, almost $50 \%$ of the wild-type activity was

Table 2 Comparison of conversions of recombinant $E$. coli and S. avermitilis for 3 '-ODI production

\begin{tabular}{lccc}
\hline Strains & $\begin{array}{l}\text { Conversion } \\
(\mathbf{\%})^{*}\end{array}$ & $\begin{array}{l}\text { Productivity } \\
(\boldsymbol{\mu} \mathbf{g} / \mathbf{h r} / \boldsymbol{g} \text { cells })\end{array}$ & $\begin{array}{l}\text { Fold- } \\
\text { change }\end{array}$ \\
\hline E. coli BL21::ASDH & 2.1 & 2.9 & 0.3 \\
Wild type S. avermitilis & 6.7 & 9.3 & 1.0 \\
$\Delta$ 3'-DH & 3.4 & 4.7 & 0.5 \\
$\Delta$ 3'-DH::3'-ASDH & 15.5 & 21.4 & 2.3 \\
Wild type::3'-DH & 22.0 & 30.5 & 3.3 \\
Wild type:: 3'-ASDH & 34.6 & 48.4 & 5.2 \\
\hline
\end{tabular}

*100 $\mu \mathrm{M}$ of substrate was used as an initial concentration at each reaction. 
retained, whereas strain $\triangle 3$ '-DH::3'-ASDH restored conversion and the conversion yield was 2.3-fold higher than that of the wild-type, suggesting that 3 '- DH is responsible for daidzein hydroxylation at the 3'-position. Further investigation of the productivity by $S$. avermitilis $\triangle 3$ '-DH::3'-ASDH strain revealed that 3'-ASDH was functionally expressed in $S$. avermitilis and could produce 3'-ODI with a conversion rate of $15.5 \%$ without any background influence by 3 '-DH in its genome. These results indicated that artificial self-sufficient fusion enzymes could work in the $S$. avermitilis recombinant strain, and its catalytic activity in terms of product production was increased by more than 4 times along with enzyme kinetic parameters.

\section{Computational structure prediction of 3 '-ASDH and verification of electron transfer distance}

In this study, daidzein was hydroxylated by oxidation catalyzed by the fusion enzyme, wherein an additional diflavin containing the reductase responsible for transfer of a reducing equivalent from NADPH to CYP heme was additionally fused including original linker sequences. Since this orientation of the electron transfer system was not native, the distance between the heme iron and flavin was very important regarding enzyme activation and coupling efficiency. Here, we used an original linker domain in the sequence of CYP102D1, so as not to change its natural folding or the flexibility of linker peptides (VRARQEHERTVFGAADLQTD). To verify electron transfer, we generated computational models of both native CYP102D1 and 3'-ASDH, and measured the heme-FMN distances.

Computational simulation revealed that the ASDH enzyme had a modular structure in which FAD/FMN and NADPH binding sites were situated distinctively at the
$\mathrm{N}$-terminal, central and C-terminal regions. As shown in Figure 5, both the heme and flavin structures were incorporated together in the protein. The flavin structure was located close to the non-native heme iron center within a calculated distance of $22.4 \AA$ (Figure 5B), which was very reasonable for the inter-molecular transfer of electrons based on the distance of $23.6 \AA$ (Figure 5A) between FMN and heme center in the native CYP102D1 enzyme structure [17]. Although the conformational changes induced by interaction between reductase and CYP or substrate were not exactly predicted or calculated, sufficient electron transfer took place and enzymatic reactions occurred very quickly. Further investigations of this CYP-flavoprotein system, its electron transfer rate, and the coupling efficiency between the reconstituted and fused systems are underway.

\section{Discussion}

In synthetic chemistry, many synthetic strategies for carbon-hydrogen $(\mathrm{C}-\mathrm{H})$ oxidation rely on a reactive intermediate that involves subtle differences in $\mathrm{C}-\mathrm{H}$ bond strength to achieve regio-selectivity. Owing to the large number of $\mathrm{C}-\mathrm{H}$ bonds in most bioactive chemicals, identifying a reagent that can specifically react at one $\mathrm{C}$ $\mathrm{H}$ bond is usually difficult. Thus, concerning regioselectivity, enzymatic oxidation catalyzed by P450s on non-activated carbon is a great challenge in modern industrial processes such as drug development and biofuel generation.

As a model system, we demonstrated the regio-specific hydroxylation of daidzein of which their biological activities or functions were greatly affected to obtain more than 30 times higher anti-oxidant capacity by hydroxylation on its 3'-position using an artificial self-sufficient enzyme [31,32]. Here, we solved the requirement for
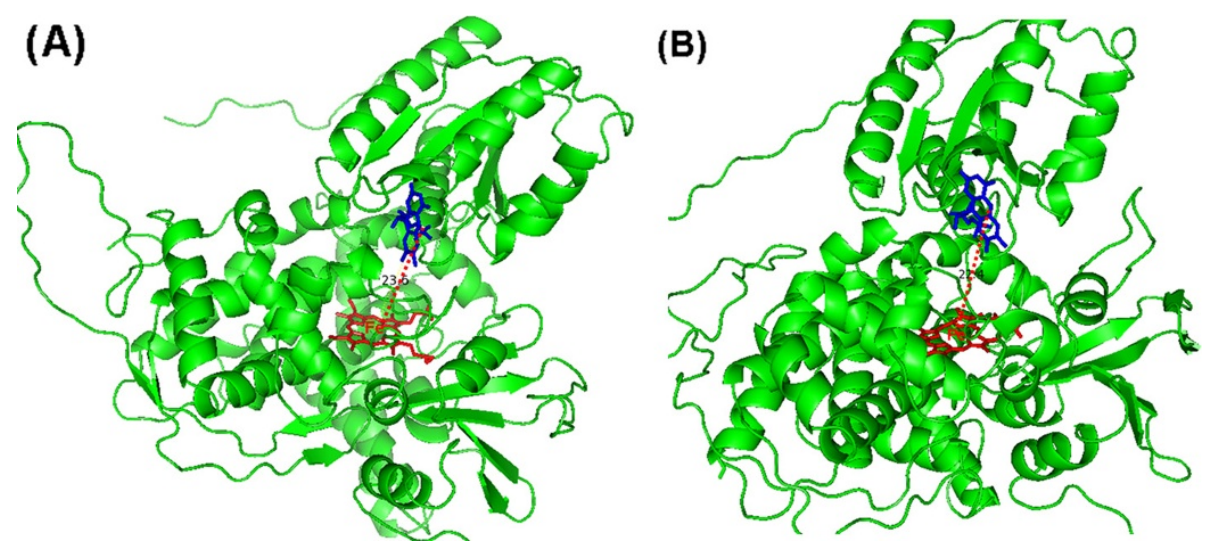

Figure 5 Computational simulation revealing coincident incorporation of heme and flavin structures in the protein. Here, the flavin structure was located close to unnative heme iron center within a calculated distance of $22.4 \AA$ (Figure 5B), which is very reasonable for the intermolecular transfer of electrons based on the distance of $23.6 \AA$ (Figure $5 \mathbf{A}$ ) between FMN and heme center in the native CYP102D1 enzyme structure. 
redox proteins, which is considered to be a bottle neck in CYP enzyme reaction, by fusion construction. As the synthetic 3'-ASDH gene was successfully expressed in $E$. coli after the codon optimization process, we were able to compare its catalytic activity with that of the 3'-DHCamAB system [25]. The fusion-enabled enzyme displayed a higher turn-over rate and higher conversion without any alterations of regio-specificity and substrate specificity, compared with the parent enzyme.

Use of $E$. coli as a biotransformation system carries many limitations. For example, the bacteria require exogenous transfer of electron from redox proteins such as CamA and CamB [30]. In addition, E. coli are very susceptible to the killing action of organic solvents used in dissolving substrate in the reaction [33,34]. As an alternative organism, Streptomyces preserves high redox potential during enzymatic reactions; the organism has proved useful for production of various antibiotics or secondary metabolites, which need many redox proteins. As well, Streptomyces is less toxically affected by organic solvents [35]. These attributes have been exploited in the use of Streptomyces as a CYP reaction host for daidzein biotransformation. Furthermore, it is possible to dissolve substrate in the reaction up to more than final volume of $1 \mathrm{mM}$ when using 10\% of DMSO:MeOH (50:50) mixed solvents without any inhibition of cell growth or cell toxicity (Table 3) [29]. With these characteristics in combination with gene manipulation systems like over-expression and gene disruption, S. avermitilis could be a strong host for biotransformation of daidzein [29].

Although S. avermitilis has been used for the heterologous expression or secretion of several polypeptides of bacterial and eukaryotic origin, heterologous genes usually need genetic modification in ribosomal binding sites or in its full DNA sequences for successful expression. However, 3'-DH and reductase domain originate from $S$. avermitilis and no genetic modifications was necessary for expression. Disruption of sav7469 (3'-DH) regarded as a major factor in 3'-ODI production and overexpression of 3'-ASDH in this deletion mutant finally proved that it is well-expressed in its original host and operates in daidzein biotransformation more efficiently as well. Although only 3'-DH was not operative in daidzein hydroxylation, considering that $S$. avermitilis $\Delta 3^{\prime}-\mathrm{DH}$ retained some activity for 3'-ODI production, the conversion of daidzein using $S$. avermitilis::3'-ASDH strain increased more than 4-times. Compared to the conversion rate of $<5 \%$ in $E$. coli biotransformation, this is an absolutely increased value. This yield is very close to the levels that are attractive for industrial applications.

This study regarding the combination of enzyme engineering and S. avermitilis host development opens up the possibility of actinomycetes utilization as a recombinant
Table 3 Comparison of conversions of recombinant Wild type:: 3'-ASDH for 3'-ODI production depending on the concentrations of DMSO

\begin{tabular}{llllll}
\hline $\begin{array}{l}\text { DMSO:MeOH } \\
(\mathbf{5 0 : 5 0 )}(\%)\end{array}$ & $\mathbf{0}$ & $\mathbf{5}$ & $\mathbf{1 0}$ & $\mathbf{2 0}$ & $\mathbf{4 0}$ \\
\hline Conversion (\%)* & 20.5 & 26.7 & 34.6 & 4.5 & n.d.
\end{tabular}

*100 $\mu \mathrm{M}$ of substrate was used as an initial concentration at each reaction. n.d. not determined.

expression host system for the production of biologically important compounds using fusion P450 proteins.

Competing interests

The authors declare that they have no competing interests.

\section{Authors' contributions}

CKY, JDH, ABR, SCM and PBP carried out the molecular genetic studies and structural analysis of samples. And JE and PHY participated in the sequence alignment and computer simulations. Finally YHD and KBG drafted and revised the full manuscript. All authors read and approved the final manuscript.

\section{Acknowledgements}

This research was supported by a National Research Foundation of Korea (NRF) grant funded by the Korean government (MEST) (No. 20090083035) and World Class University Program (R322009000102130).

\section{Author details}

${ }^{1}$ School of Chemical and Biological Engineering, Institute of Bioengineering, Seoul National University, Seoul, South Korea. ${ }^{2}$ School of Biotechnology, Yeungnam University, Gyeongsan, Gyeongbuk, South Korea. ${ }^{3}$ Interdisciplinary Program for Bioengineering, Seoul National University, Seoul 151-744, Republic of Korea. ${ }^{4}$ Korea Bio-Hub Center, Bio-MAX Institute, Seoul National University, Seoul, South Korea.

Received: 9 March 2012 Accepted: 16 May 2012

Published: 14 June 2012

\section{References}

1. Yun $\mathrm{CH}$, Kim KH, Kim DH, Jung HC, Pan JG: The bacterial P450 BM3: a prototype for a biocatalyst with human P450 activities. Trends Biotechnol 2007, 25:289-298.

2. Astrid S: HS, Roland S: Diversities and similarities in P450 systems: An introduction. Met lons Life Sci 2007, 3:1-3.

3. Huttunen KM, Mahonen N, Raunio H, Rautio J: Cytochrome P450-activated prodrugs: targeted drug delivery. Curr Med Chem 2008, 15:2346-2365.

4. Urlacher VB, Girhard M: Cytochrome P450 monooxygenases: an update on perspectives for synthetic application. Trends Biotechnol 2012, 30:26-36.

5. Bernhardt R: Cytochrome P450: structure, function, and generation of reactive oxygen species. Rev Physiol Biochem Pharmacol 1996, 127:137-221.

6. Munro AW, Girvan HM, McLean KJ: Cytochrome P450-redox partner fusion enzymes. Biochim Biophys Acta 2007, 1770:345-359.

7. O'Reilly E, Kohler V, Flitsch SL, Turner NJ: Cytochromes P450 as useful biocatalysts: addressing the limitations. Chem Commun (Camb) 2011, 47:2490-2501.

8. Hannemann F, Bichet A, Ewen KM, Bernhardt R: Cytochrome P450 systems-biological variations of electron transport chains. Biochim Biophys Acta 2007, 1770:330-344.

9. Roberts GA, Grogan G, Greter A, Flitsch SL, Turner NJ: Identification of a new class of cytochrome P450 from a Rhodococcus sp. J Bacteriol 2002, 184:3898-3908.

10. De Mot R, Parret AH: A novel class of self-sufficient cytochrome P450 monooxygenases in prokaryotes. Trends Microbiol 2002, 10:502-508.

11. Gustafsson MC, Roitel O, Marshall KR, Noble MA, Chapman SK, Pessegueiro A, Fulco AJ, Cheesman MR, von Wachenfeldt C, Munro AW: Expression, purification, and characterization of Bacillus subtilis cytochromes P450 
CYP102A2 and CYP102A3: flavocytochrome homologues of P450 BM3 from Bacillus megaterium. Biochemistry 2004, 43:5474-5487.

12. Budde M, Maurer SC, Schmid RD, Urlacher VB: Cloning, expression and characterisation of CYP102A2, a self-sufficient P450 monooxygenase from Bacillus subtilis. Appl Microbiol Biotechnol 2004, 66:180-186.

13. Chowdhary PK, Alemseghed M, Haines DC: Cloning, expression and characterization of a fast self-sufficient P450: CYP102A5 from Bacillus cereus. Arch Biochem Biophys 2007, 468:32-43.

14. Dietrich M, Eiben S, Asta C, Do TA, Pleiss J, Urlacher VB: Cloning, expression and characterisation of CYP102A7, a self-sufficient P450 monooxygenase from Bacillus licheniformis. Appl Microbiol Biotechnol 2008, 79:931-940.

15. Choi KY, Jung E, Jung DH, Pandey BP, Yun H, Park HY, Kazlauskas RJ, Kim BG: Cloning, expression and characterization of CYP102D1, a selfsufficient P450 monooxygenase from Streptomyces avermitilis. FEBS $J$ 2012, 279:1650-1662.

16. Loida PJ, Sligar SG: Molecular recognition in cytochrome P-450: mechanism for the control of uncoupling reactions. Biochemistry 1993, 32:11530-11538

17. Noble MA, Miles CS, Chapman SK, Lysek DA, Mackay AC, Reid GA, Hanzlik RP, Munro AW: Roles of key active-site residues in flavocytochrome P450 BM3. Biochem J 1999, 339(Pt 2):371-379.

18. Dodhia VR, Fantuzzi A, Gilardi G: Engineering human cytochrome P450 enzymes into catalytically self-sufficient chimeras using molecular Lego. J Biol Inorg Chem 2006, 11:903-916.

19. Nodate M, Kubota M, Misawa N: Functional expression system for cytochrome P450 genes using the reductase domain of self-sufficient P450RhF from Rhodococcus sp. NCIMB 9784. Appl Microbiol Biotechnol 2006, 71:455-462.

20. Pandey BP, Roh C, Choi KY, Lee N, Kim EJ, Ko S, Kim T, Yun H, Kim BG: Regioselective hydroxylation of daidzein using P450 (CYP105D7) from Streptomyces avermitilis MA4680. Biotechnol Bioeng 2010, 105:697-704.

21. Lamb DC, Ikeda H, Nelson DR, Ishikawa J, Skaug T, Jackson C, Omura S, Waterman MR, Kelly SL: Cytochrome p450 complement (CYPome) of the avermectin-producer Streptomyces avermitilis and comparison to that of Streptomyces coelicolor A3(2). Biochem Biophys Res Commun 2003, 307:610-619.

22. Zhao $X$, Wang $Y$, Wang S, Chen Z, Wen Y, Song Y: Construction of a doramectin producer mutant from an avermectin-overproducing industrial strain of Streptomyces avermitilis. Can J Microbiol 2009, 55:1355-1363.

23. Ikeda H, Ishikawa J, Hanamoto A, Shinose M, Kikuchi H, Shiba T, Sakaki Y, Hattori M, Omura S: Complete genome sequence and comparative analysis of the industrial microorganism Streptomyces avermitilis. Nat Biotechnol 2003, 21:526-531.

24. Hong YS, Lee JH, Kim HS, Kim KW, Lee JJ: Targeted gene disruption of the avermectin B O-emthyltransferase gene in Streptomyces avermitlis. Biotechnol Lett 2001, 23:1765-1770.

25. MacNeil DJ, Klapko LM: Transformaton of Streptomyces avermitilis by plasmid DNA. J Ind Microbiol 1987, 2:209-218.

26. Omura T, Sato R: The Carbon Monoxide-Binding Pigment of Liver Microsomes. I. Evidence for Its Hemoprotein Nature. J Biol Chem 1964, 239:2370-2378.

27. Xu LH, Fushinobu S, Takamatsu S, Wakagi T, Ikeda H, Shoun H: Regio- and stereospecificity of filipin hydroxylation sites revealed by crystal structures of cytochrome P450 105P1 and 105D6 from Streptomyces avermitilis. J Biol Chem 2010, 285:16844-16853.

28. Li HY, Darwish K, Poulos TL: Characterization of recombinant Bacillus megaterium cytochrome P-450 BM-3 and its two functional domains. J Biol Chem 1991, 266:11909-11914.

29. Roh C, Seo SH, Choi KY, Cha M, Pandey BP, Kim JH, Park JS, Kim DH, Chang IS, Kim BG: Regioselective hydroxylation of isoflavones by Streptomyces avermitilis MA-4680. J Biosci Bioeng 2009, 108:41-46.

30. Pandey BP, Lee N, Choi KY, Jung E, Jeong DH, Kim BG: Screening of bacterial cytochrome P450s responsible for regiospecific hydroxylation of (iso)flavonoids. Enzyme Microb Technol 2011, 48:386-392.

31. Ososki AL, Kennelly EJ: Phytoestrogens: a review of the present state of research. Phytother Res 2003, 17:845-869.

32. Park JS, Kim DH, Shim JS, Kim JS, Choi KY, Kim BG, Kim DH, Kim HK: 7,8,4'Trihydroxyisoflavone from Fermented Soybean Food and Its Biological Activity. J Soc Cosmet Scientists Korea 2010, 36(3):221-226.
33. Sardessai $Y$, Bhosle S: Tolerance of bacteria to organic solvents. Res Microbiol 2002, 153:263-268.

34. Aono R, Nakajima H: Organic solvent tolerance in Escherichia coli. Tanpakushitsu Kakusan Koso 1997, 42:2532-2541.

35. Chen G, Wang GY, Li X, Waters B, Davies J: Enhanced production of microbial metabolites in the presence of dimethyl sulfoxide. J Antibiot (Tokyo) 2000, 53:1145-1153.

doi:10.1186/1475-2859-11-8

Cite this article as: Choi et al:: Engineering of daidzein 3'-hydroxylase P450 enzyme into catalytically self-sufficient cytochrome P450. Microbial Cell Factories 2012 11:81.

\section{Submit your next manuscript to BioMed Central and take full advantage of:}

- Convenient online submission

- Thorough peer review

- No space constraints or color figure charges

- Immediate publication on acceptance

- Inclusion in PubMed, CAS, Scopus and Google Scholar

- Research which is freely available for redistribution 